\title{
Damit zusammenwächst, was zusammengehört
}

\author{
Helmut Kreidenweis
}

\begin{abstract}
Die Informationstechnologie ist inzwischen in vielen Unternehmen auch der Sozialwirtschaft die Basis vitaler Geschäftsprozesse. Daher macht das oft mangelhafte Zusammenwirken verschiedener Programme und Computer - noch dazu angesiedelt in verschiedenen Diensten und Einrichtungen - große Probleme. Die Software- und DatenIntegration ist deshalb mehr als ein Thema nur für die EDV-Verantwortlichen.
\end{abstract}

»Die integrierte Software-Lösung für Ihre Einrichtung. « Flotte Floskeln wie diese zieren heute fast alle Hochglanzprospekte der großen Branchensoftware-Anbieter. Und doch suchen viele Komplexeinrichtungen oder Verbände noch immer vergeblich nach geeigneten Systemen. Bleibt also die »eierlegende Wollmilchsau « pure Fiktion? Vielleicht. Doch mittlerweile gibt es in der Welt der Informationstechnologie (IT) Entwicklungen, die hoffen lassen.

Wer sich die Software-Ausstattung vieler Einrichtungen und Verbände ansieht, fühlt sich an einen bunten Flickenteppich erinnert: Klientenverwaltung, Rechnungswesen, Personalwirtschaft, Controlling, Dienstplanung oder Materialwirtschaft sind auf mehr oder weniger viele Einzelprodukte unterschiedlicher Hersteller verteil. Ein ebenso buntes Häufchen an Datenbanken und Datenformaten auf Servern und Arbeitsplatz-Computern gesellt sich hinzu.

In der Folge werden Klienten- und Mitarbeiter-Daten oft mehrfach vorgehalten und mühsam von Hand oder mit wackeligen Schnittstellen übertragen. Das Controlling sammelt diese Daten irgendwann wieder zusammen, strickt daraus monströse Ex-
cel-Tabellen und kann trotzdem nicht die Informationen liefern, die für eine zeitnahe Steuerung benötigt werden. Auch Geschäftsprozesse wie etwa die Klientenaufnahme können wegen der vielen Medienbrüche nicht durchgängig in Software abgebildet und an einem Arbeitsplatz zusammengefasst werden.

\section{Wege zur Integration}

Bis heute wird oft versucht, das Integrationsproblem durch Schnittstellen zu lösen. Diese Zusatzprogramme bereiten Daten so auf, dass sie sich in die Speicher- und Verarbeitungslogik anderer Programme einpassen. Wird bei einem der beteiligten Programme die Datenstruktur umgestellt, so legt dies die Schnittstellen oft für Wochen oder Monate lahm. Der Schnittstellen-Programmierer muss sich die Informationen zur neuen Datenstruktur oft mühsam beschaffen, die Informationspolitik der Firmen ist hier oft wenig rühmlich. Meist ist über Schnittstellen auch kein Echtzeit-Austausch möglich, die Daten stehen erst nach Stunden oder am nächsten Tag in der jeweils anderen Software zur Verfügung. Schnittstellen mit eingebauter Verarbeitungslogik, die beispielsweise Doppelungen oder Ausnahmefälle erkennen, sind bislang eher die Ausnahme und in der Regel auf zentrale Produkte gut kooperierender Hersteller beschränkt.

Als Antwort auf die SchnittstellenProblematik und als Königsweg zur Integration wurde vor allem in der gewerblichen Wirtschaft die Entwicklung sogenannter ERP-Systeme (Enterprise Ressource Planning) propagiert: Alle betriebswirtschaftlichen Funktionen werden in einer Software auf der Grundlage einer Datenbank abgebildet. Jeder Kunde, jedes Pro-

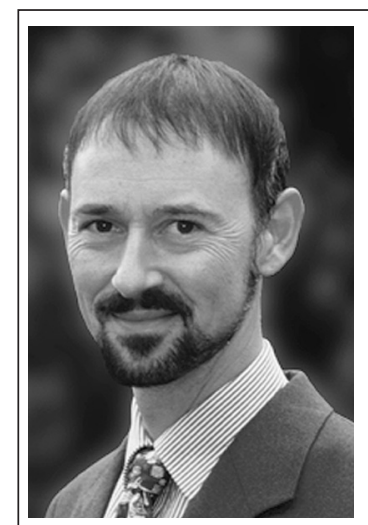

Helmut
Kreiden-
weis ist
Professor
für Sozial-
informa-
tik an der
Katholi-
schen
Univer-
sität
Eichstätt-
Ingol-

stadt und Inhaber der Beratungsfirma KI-Consult, Augsburg. Er ist Mitveranstalter der Fachmesse ConSozial und Mitglied im Beirat der Zeitschrift SOZIALwirtschaft und des Informationsdienstes SOZIALwirtschaft aktuell. E-Mailhk@ki-consult.de

dukt oder jeder Mitarbeiter ist nur singulär im System repräsentiert, Prozesse können durchgängig gestaltet werden und das Controlling kann ohne Systembrüche darauf zugreifen. So weit die Theorie (vgl. Grafik ERPBeispielkonzept auf Seite 10).

In der Praxis stellte sich immer wieder heraus, dass die betriebliche Realität in den meisten Branchen viel zu facettenreich und dynamisch ist, als dass sie durchgängig in einem System abgebildet werden könnte. Zwar bauten die Hersteller ihre ERP-Software mehr und mehr aus, doch je weiter diese Software-Schlachtschiffe wuchsen, um so träger und fehleranfälliger wurden sie.

Um die Jahrtausendwende begann sich deshalb die Strategie zu ändern: Global agierende IT-Anbieter ersannen zunächst vor allem für Großkon- 
zerne neue Software-Architekturen, die sich als intelligente Agenten zwischen die unterschiedlichen Einzelanwendungen schalten und den Datenverkehr nach flexiblen

Regelsystemen steuern. Mit Konzepten wie Middleware oder Enterprise Application Integration (EAI) können beispielsweise in den angeschlossenen Programmen selbstständig neue Datensätze generiert, die Anlage von Daten-Dubletten verhindert und Geschäftsprozesse durchgängig abgebildet werden. Die unter der Integrationsschicht liegenden Programme bleiben dabei für den Normalanwender unsichtbar, die Daten werden für ihn zumeist auf einer web-basierten Oberfläche zusammengeführt. Der Datenzugriff ist daher oft auch betriebssystem- und arbeitsplatzunabhängig möglich.

\section{IT-Integration in der Sozialwirt- schaft}

In der Sozialwirtschaft wurde die Notwendigkeit zur IT-Integration häufig erst im Zuge der Herausbildung von Konzern- oder Holdingstrukturen oder anlässlich von Fusionen und Übernahmen erkannt. Auch begannen manche Vorstände und Geschäftsführer angesichts enger werdender Finanzspielräume schmerzlich $\mathrm{zu}$ realisieren, dass es in den bisherigen Systemen kaum möglich ist, jenseits der Finanzbuchhaltung differenzierte und zeitnahe Steuerungsinformationen zu bekommen.

Zwar nahm und nimmt die ITDurchdringung in pflegerischpädagogischen, haus- oder gebäudewirtschaftlichen Bereichen stetig zu, doch ist dies meist mit der Einführung hochgradig spezialisierter Software-Lösungen verbunden, die untereinander und mit den vorhandenen Systemen nicht oder nur über Schnittstellen kommunizieren können.

Größere Einrichtungen oder Verbände mit eigenen Einrichtungsstrukturen verfügen zumeist über ein breites Portfolio an Fachbereichen, die jeweils durch ganz eigene Geschäftslogiken geprägt sind. Auf der Basis moderner Organisationskonzepte erweist sich hier häufig ein Mix aus zentralen und dezentralen Elementen der Informationsverarbeitung und Steuerung als sinnvoll. Backoffice-

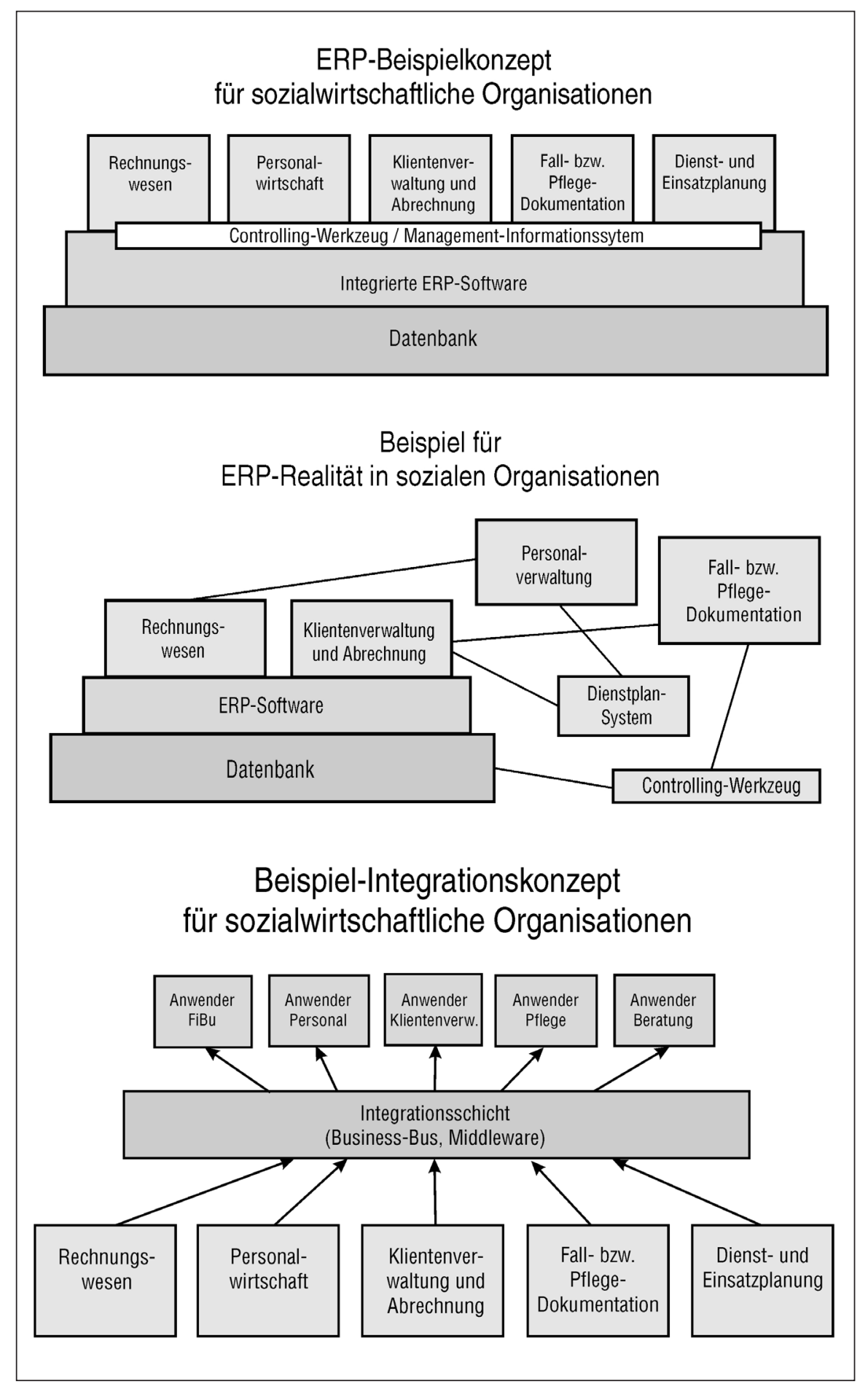

Funktionen wie Finanzbuchhaltung, Controlling oder Personalwirtschaft sollen vielfach in der Zentrale gebündelt werden. Frontoffice-Funktionen wie Klientenverwaltung, Dienstplanung oder Dokumentation werden in den Einrichtungen angesiedelt, also dort, wo diese Daten entstehen und täglich gebraucht werden. Die Haltung der Daten soll jedoch auch in diesem Falle zentral erfolgen.

Als erste haben sich große Komplexeinrichtungen auf den Weg zur Integration gemacht. Der Preis dafür ist nicht selten hoch: Vielfach setzen sie auf teure ERP-Lösungen aus der gewerblichen Wirtschaft, die mit hohem Kostenaufwand an die Spezifika der Sozialwirtschaft angepasst werden und dennoch in vielen Teilfunktionen auf Schnittstellen zu Spezialsoftware angewiesen sind (vgl. Grafik oben: ERP-Realität).

Viele andere Träger sind zwar latent unzufrieden, haben sich jedoch noch nicht auf diesen steinigen Pfad begeben. Ihnen ist häufig gemeinsam, dass sie noch über wenig ausgeprägte 
Managementstrukturen und selten über eine strategische IT-Planung verfügen.

Entsprechend stellt sich auch der Angebotsmarkt dar: Seit über 20 Jahren herrscht Arbeitsteilung zwischen Anbietern für sozialwirtschaftliche Fachsoftware und betriebswirtschaftliche Standardsoftware. Manche Anbieter haben zwar Rechnungswesen und Klientenverwaltung in RumpfERP-Systemen integriert, jedoch fehlt es hier oft an anderer Stelle: Funktionen wie Personalverwaltung, Dienstplanung oder Controlling müssen weiterhin über proprietäre Schnittstellen angebunden werden.

So ist bislang kein Anbieter in der Lage, voll integrierte und durchgängig auf modernen Software-Architekturen basierende Systeme zu liefern, die gleichzeitig alle betriebswirtschaftlichen Aufgaben erfüllen und tigen Software-Weltkonzernen mit ihrer Programmier- und Vertriebsarmada aufgemischt wird?

In der gewerblichen Wirtschaft gilt die SAP AG mit einem Weltmarktanteil von über 20 Prozent und 33.000 Unternehmenskunden längst als unangefochtener Spitzenreiter bei ERPSystemen und betriebswirtschaftlicher Datenintegration. Seine Systeme R/3 und mySAP, später ergänzt durch die Mittelstandslösungen Business One (bis 100 Mitarbeiter) und mySAP All in One (bis 2.500 Mitarbeiter) versprachen den Unternehmen eine hoch integrierte Datenhaltung und durchgängige Abbildung der Geschäftsprozesse. Doch im Zuge der wachsenden Durchdringung aller Unternehmensbereiche mit IT musste SAP erkennen, dass selbst ein Platzhirsch mit einem großen Rudel an Partnerfirmen nicht in der Lage ist, in allen Winkeln der Wirtschaft Spitzen-

\section{»Der Datenaustausch über Schnittstellen ist mühsam und fehleranfällig «}

die ganze Palette der fachlichen und administrativen Funktionen der Sozialwirtschaft in der gebotenen Tiefe abdecken. Auch ist der Anbietermarkt mit mehrern hundert, meist auf den deutschsprachigen Markt beschränkten Herstellern viel zu zersplittert, als dass sich entsprechend starke Wettbewerber bislang hätten positionieren können.

So erklärt es sich vielleicht auch, dass sich die gängigen FachsoftwareAnbieter in der Sozialwirtschaft den ERP-Begriff bisher nicht so recht auf die Fahnen zu schreiben getraut haben, obgleich er auch in manch anderen Branchen stillschweigend und konträr zur ursprünglichen Intention - auf betriebswirtschaftliche Kernfunktionen reduziert wurde.

\section{SAP und Microsoft: Welche Rolle spielen die Global Player?}

Höchste Zeit also, dass die mittelalterliche Kleinstaaterei von den mäch- lösungen anzubieten. Vor einigen Jahren ersann man daher in Walldorf eine neue Strategie mit Namen »Netweaver «: SAP wollte nicht mehr »nur« Lösungsanbieter sein, sondern im Sinne des oben genannten EAIKonzeptes der Integrator für Programme verschiedener Anbieter.

In der Sozialwirtschaft ist davon jedoch noch nicht viel zu sehen. Die Marktdurchdringung mit SAP-Systemen ist hier eher gering und zumeist auf Komplexeinrichtungen mit deutlich über 1.000 Mitarbeitern beschränkt. SAP hat sich im Übrigen bislang auch nicht selbst um diesen Markt gekümmert, sondern dieses "Kleinvieh « gerne seinen Partnern überlassen.

Und wie ist es um den Software-Giganten aus Redmond bestellt? Seit der Übernahme der betriebswirtschaftlichen Software Navision im Jahr 2002 versucht auch Microsoft, der Übermacht von SAP auf dem Markt für Unternehmenssoftware etwas entgegen zu setzen. Doch Navisi- on, seit kurzem unter dem Namen Microsoft Dynamics NAV vertrieben, ist bislang primär in kleinen und mittleren Unternehmen im Einsatz und das für Großunternehmen gedachte, bislang eigenständige ERPProdukt Axapta spielt hierzulande kaum eine Rolle.

In der Sozialwirtschaft tauchte Microsoft bislang nur in Gestalt variantenreicher Partner-Konstellationen auf. Mittlerweile scheint sich das Unternehmen jedoch auch selbst in das Geschäftsfeld einzuklinken. Offensichtlich hat man inzwischen die Potenziale dieses Marktes mit über 1,2 Million Beschäftigten und 70 Milliarden Euro Jahresumsatz erkannt. Doch zeichnet sich ein von der SAP-Strategie abweichender Ansatz ab: In nicht ganz untypischer Microsoft-Manier betont man, dass Partner willkommen sind - jedoch nur, wenn Sie Ihre Software auf der technologischen Basis von Navision entwickeln. Ob und in welchen Zeiträumen Microsoft mit dieser Strategie erfolgreich sein wird, bleibt abzuwarten. Angesichts einer gewachsenen Software-Landschaft mit unterschiedlichen technologischen Historien und teils weit entwickelter, hoch differenzierter Branchensoftware dürfte es jedoch nicht leicht sein, die Anbieter von Branchenlösungen und am Ende auch die breite Masse von Anwendern von diesem Weg zu überzeugen.

\section{Ausblicke}

Wohin führt also der Integrationspfad der Zukunft? So lange die Sozialwirtschaft die Daten- und SoftwareIntegration nicht mit Nachdruck vom Markt einfordert, wird sich an der Situation der letzten 20 Jahre strukturell wenig ändern. Zwar versprechen moderne serviceorientierte Software-Architekturen mit Web Services oder systemübergreifenden Datenformaten wie XML auf längere Sicht mehr Durchlässigkeit. Doch gerade in den großen Programmen großer Anbieter steckt oft noch jede Menge alter Datencode, der die Nutzung solcher Technologien erschwert oder gar verhindert.

Als pragmatischer und mittelfristig sinnvollster Weg erscheint derzeit eine Integrationsstrategie auf der Basis von Middleware-Produkten. Aus welcher Software-Schmiede diese Pro- 
dukte freilich kommen können oder sollen, ist eine offene Frage. Denn der Middleware-Anbieter ist immer auch die Spinne im Netz, die die Fäden zusammenhält (vgl. Grafik auf Seite 10 »Beispiel-Integrationskonzept für sozialwirtschaftliche Organisationen «).

Für die Anwender nutzbringend wäre in jedem Fall eine engere Zusammenarbeit der Branchensoftware-Anbieter im deutschsprachigen Raum. Wenn diese bereit sind, sich von der Illusion zu verabschieden, die im Sozialmarkt benötigte Funktionalität bis in den letzten Winkel selbst optimal abbilden zu können, steht dem nichts mehr im Wege. Immerhin: Auf einer im März 2006 an der Katholischen Universität Eichstätt veranstalteten Fachtagung zeigten auch viele Software-Anbieter Interesse an einem regelmäßigen Dialog untereinander und - jenseits der üblichen Akquise- und Kundenkontakte - auch mit der Sozialwirtschaft und Wissenschaft. Ein Ziel der neu geschaffene Professur für Sozialinformatik an dieser Hochschule ist es, diesen Dialog zum Nutzen aller beteiligter weiter $\mathrm{zu}$ fördern und kritisch zu begleiten.

\section{Wissen, was man weiß}

Täglich verarbeiten soziale Organisationen Informationen aller Art, zur Erfüllung ihrer Aufgaben benötigen sie vielfältiges Wissen. In Zeiten verschärften Wettbewerbs entscheiden Tempo und Qualität der Nutzung neuer Erkenntnisse zunehmend über Bestand und Erfolg. Wissen ist zu einem zentralen Produktionsfaktor für soziale Dienstleister geworden. Doch der Umgang mit ihm wird bislang kaum gesteuert. Hier setzt das Wissensmanagement an: Sein Ziel ist es, vorhandenes Wissen besser zu nutzen und neue Wissensressourcen $\mathrm{zu}$ erschließen. Ein neues Buch aus der Reihe "Studienkurs Management in der Sozialwirtschaft« zeigt, welche Arten von Wissen es gibt und was beim Management von Wissen in sozialen Organisationen berücksichtigt werden muss. Die Kernprozesse des Wissensmanagements werden ebenso verständlich dargestellt wie Strategien und Methoden zur Einführung. Der informationstechnologischen Unterstützung widmet sich ein eigenes Kapitel.

Helmut Kreidenweis/Willi Steincke: Wissensmanagement. Nomos Verlagsgesellschaft, Baden-Baden 2005. 161 Seiten. 19,80 Euro. ISBN 3-8329-1613-X.

\section{Tipps für Führungskräfte, IT-Ressoucen effizient zu vergeuden}

1 Informationstechnologie ist ein Fachgebiet für Techniker.

1. Führungskräfte und Sozialarbeiter haben besseres zu tun und sollten sich nicht in Dinge einmischen, von denen sie nichts verstehen.

2 Weisen Sie ihren EDV-Verantwortlichen in die Schranken. Er hat . sich auf seinen Schraubenzieher zu konzentrieren und sich darum zu kümmern, dass die Computer laufen. Arbeitsprozesse in der Verwaltung oder gar in der Sozialarbeit gehen ihn nichts an.

3 Dezentralisieren Sie konsequent Verantwortung: Wenn ein Mitar3. beiter einen günstigen PC oder Drucker im Supermarkt findet, soll er sofort zuschlagen. Lieber heute Geld gespart, als morgen komplizierte Lieferantenverträge ausgehandelt. Und wegen dem bisschen mehr an Installations- und Wartungsaufwand sollen die IT-Leute nur nicht anfangen zu meckern.

\section{»Vertrauen Sie einfach auf Ihr Gefühl «}

I Fordern Sie ihre Mitarbeiter: Mit PC und Software muss jeder 4. schon selbst zurechtkommen. Schulungen sind bei den heute ja so kinderleicht zu bedienenden Programmen völlig überflüssig.

5 Software-Kauf ist Vertrauenssache. Ist der Verkäufer nett, ist auch 5 . seine Software ok. Aufwendige Bedarfsanalysen sind nur Zeitvergeudung. Vertrauen Sie einfach auf Ihr Gefühl!

6 Engagieren Sie niemals IT-Berater. Die kosten nur Geld und er6. zählen Ihnen seltsame Geschichten von IT-Management und Mitarbeiter-Support, die noch mehr Geld kosten als der Berater selbst.

7 Ändern Sie keine bewährten Abläufe, nur weil neue Software ein- gesetzt werden soll. Fordern Sie vielmehr von den Programmen, dass sie alle Arbeitsabläufe exakt so abbilden, wie sie schon seit jeher waren. Alles andere bringt nur Unruhe in ihre Organisation und das ist bekanntlich schädlich.

Q Glauben Sie nicht an das Gerede von integrierter Software. Wenn 8. ihr Controller schlau genug ist, wird er die Daten für das Berichtswesen schon irgendwie zusammenklauben. Und ein Jährchen Zeitverzug im Berichtswesen hat bislang noch niemandem geschadet.

Q Gestatten Sie Ihren Mitarbeitern niemals Messen oder Software- Präsentationen zu besuchen. Sie liegen ihnen hinterher nur mit Fantasien über irgendwelche angeblich effizienz- oder qualitätssteigernde IT-Lösungen in den Ohren, anstatt sich auf ihre Arbeit zu konzentrieren.

1 Zeigen Sie Verständnis für Mitarbeiter und Führungskräfte, die 10. nicht mit dem PC arbeiten wollen. Jeder soll schließlich mit seinem persönlichen Arbeitsstil glücklich werden. Und die zehn oder fünfzehn Jahre, bis diese Kollegen ausgeschieden sind, lassen sich auch noch abwarten. 